Supporting Information for

\title{
Two-Dimensional Gold Halides: Novel Semiconductors with Giant Spin-Orbit Splitting and Tunable Optoelectronic Properties
}

Artem V. Kuklin ${ }^{a, b},{ }^{*}$, Lingfeng $\mathrm{Gao}^{c},{\text { Han } Z \mathrm{Zhang}^{c} \text { and Hans Ågren }}^{b, d, e}$

${ }^{a}$ Division of Science and Innovations, Siberian Federal University, 79 Svobodny pr., Krasnoyarsk 660041, Russia

${ }^{b}$ Department of Physics and Astronomy, Uppsala University, Box 516, SE-751 20 Uppsala, Sweden

'Institute of Microscale Optoelectronics, Collaborative Innovation Centre for Optoelectronic Science \&

Technology, Key Laboratory of Optoelectronic Devices and Systems of Ministry of Education and

Guangdong Province, College of Physics and Optoelectronic Engineering, Shenzhen Key Laboratory of

Micro-Nano Photonic Information Technology, Guangdong Laboratory of Artificial Intelligence and Digital

Economy (SZ), Shenzhen University, Shenzhen, 518060, P. R. China

${ }^{d}$ College of Chemistry and Chemical Engineering, Henan University, Kaifeng, Henan 475004 P. R. China

eTomsk State University, 36 Lenin Avenue, Tomsk, Russia

${ }^{*}$ E-mail: artem.icm@gmail.com

\section{Internal coordinates of optimized structures:}

1) bulk $\mathrm{AuBr}$ and $\mathrm{AuI}$

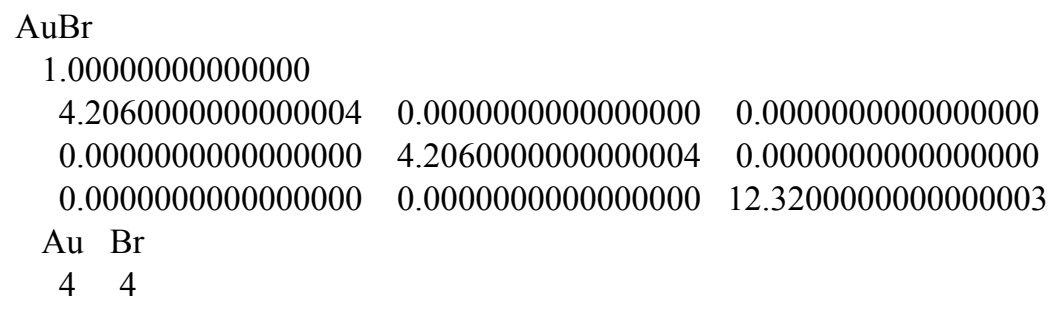

$\mathrm{AuI}$

1.00000000000000 


$\begin{array}{ccc}4.2999999999999998 & 0.0000000000000000 & 0.0000000000000000 \\ 0.0000000000000000 & 4.2999999999999998 & 0.0000000000000000 \\ 0.0000000000000000 & 0.0000000000000000 & 13.8800000000000008\end{array}$

$\mathrm{Au} \mathrm{I}$

44

Direct

$0.0000000000000000 \quad 0.0000000000000000 \quad 0.0000000000000000$

$\begin{array}{lllll}0.4999999999310347 & 0.4999999999310347 & 0.0000000000000000\end{array}$

$\begin{array}{lllll}0.4999999999310347 & 0.0000000000000000 & 0.4999999999927169\end{array}$

$\begin{array}{lllll}0.0000000000000000 & 0.4999999999310347 & 0.4999999999927169\end{array}$

$\begin{array}{llllll}0.2499999999655174 & 0.2499999999655174 & 0.1547339670016177\end{array}$

$\begin{array}{lllll}0.7499999998965521 & 0.7499999998965521 & 0.8452660330567036\end{array}$

$\begin{array}{lllll}0.2499999999655174 & 0.2499999999655174 & 0.6547339669942779\end{array}$

$\begin{array}{llll}0.7499999998965521 & 0.7499999998965521 & 0.3452660329910921\end{array}$

\section{2) single-layer $\mathrm{AuBr}$ and $\mathrm{AuI}$}

$\mathrm{AuBr}$

1.00000000000000

$\begin{array}{llll}4.1285000000000007 & 0.0445000000000000 & 0.0000000000000000\end{array}$

$\begin{array}{llll}0.0445000000000000 & 4.1285000000000007 & 0.0000000000000000\end{array}$

$\begin{array}{llll}0.0000000000000000 & 0.0000000000000000 & 20.3199996947999999\end{array}$

$\mathrm{Au} \mathrm{Br}$

22

Direct

$0.5000000000000000-0.0000000000000000 \quad 0.3031492866641656$

$\begin{array}{llll}-0.0000000000000000 & 0.5000000000000000 & 0.3031492866641656\end{array}$

$\begin{array}{llll}0.2500000000000000 & 0.2500000000000000 & 0.4036473169103483\end{array}$

$\begin{array}{llll}0.7500000000000000 & 0.7500000000000000 & 0.2026525049918761\end{array}$

$\mathrm{AuI}$

1.00000000000000

$\begin{array}{llll}4.2410000000000005 & 0.0880000000000000 & 0.0000000000000000\end{array}$

$\begin{array}{llll}0.0880000000000000 & 4.2410000000000005 & 0.0000000000000000\end{array}$

$\begin{array}{llll}0.0000000000000000 & 0.0000000000000000 & 20.3199996947999999\end{array}$

$\mathrm{Au} \mathrm{I}$

22

Direct

$0.5000000000000000-0.0000000000000000 \quad 0.3031492866641656$

$\begin{array}{llll}0.0000000000000000 & 0.5000000000000000 & 0.3031492866641656\end{array}$

$\begin{array}{llll}0.2500000000000000 & 0.2500000000000000 & 0.4113391118008068\end{array}$

$\begin{array}{llll}0.7500000000000000 & 0.7500000000000000 & 0.1949607101014189\end{array}$ 


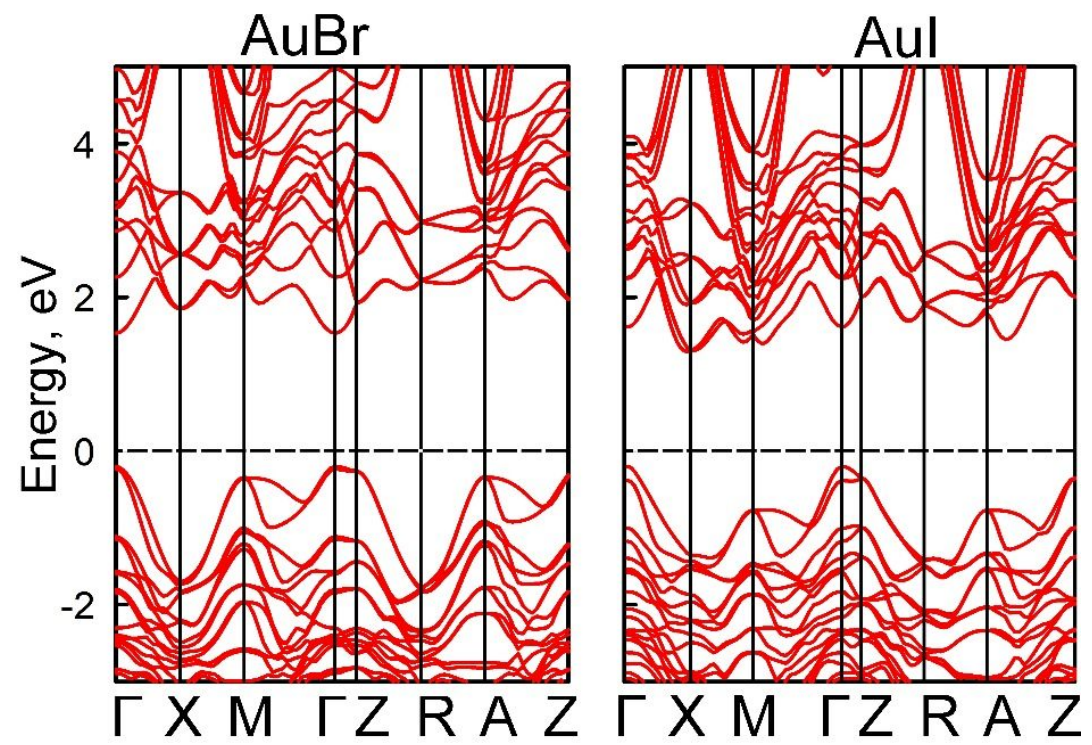

Figure S1. Band structures of bulk AuBr (left) and AuI (right) calculated by HSE06 with SOC. The Fermi energy level is set to $0 \mathrm{eV}$.
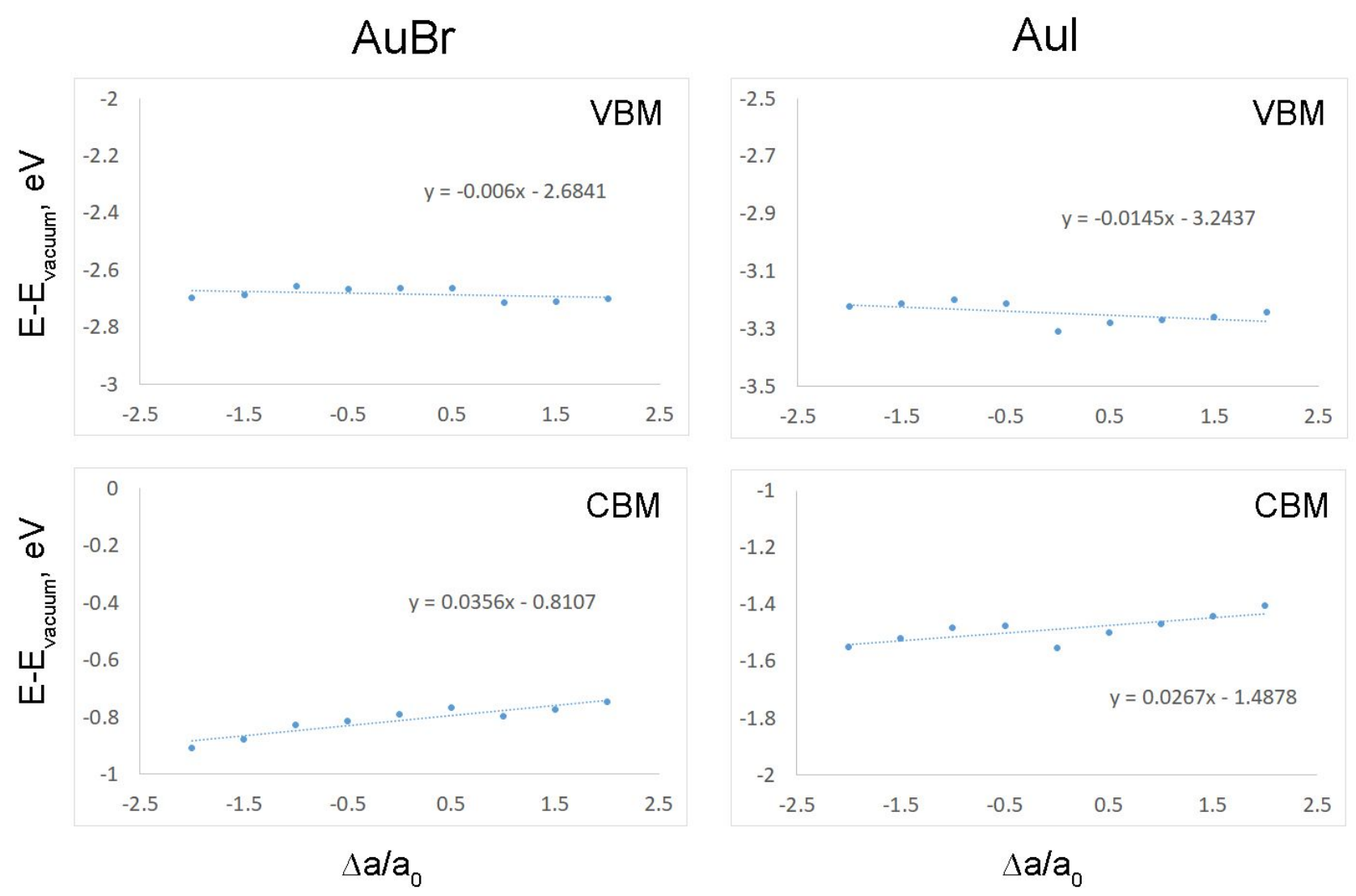

Figure S2. Linear fitting of deformation potential for 2D AuBr (left) and AuI (right). 